\title{
La importancia de las ciencias sociales contemporáneas en América Latina'
}

Artículos originales: SOCIOLOGÍA

Recibido: 11/09/2020

Aprobado: 04/11/2020

Publicado: 05/02/2021
Julio Mejía Navarrete

Universidad Nacional Mayor de San Marcos

jmejian@unmsm.edu.pe

\section{RESUMEN}

El artículo analiza el proceso de originalización teórica de América Latina que anuncia la exploración de otro sentido de la historia, ungido en la unidad de las ciencias sociales, el desarrollo de nuevas problemáticas de investigación y los desarrollos de discursos alternativos.

Palabras clave: Otro sentido de la historia; unidad de las ciencias sociales; investigación social; pensamiento latinoamericano.

\section{The importance of contemporary social sciences in Latin America}

ABSTRACT

The article analyzes the process of theoretical originalization in Latin America that announces the exploration of another sense of history, anointed in the unity of the social sciences, the development of new research problems and the development of alternative discourses.

KeYwords: Another sense of history; unity of the social sciences; social research; Latin American thought.

1 Exposición realizada en el marco del XXVI aniversario de la Facultad de Ciencias Sociales, 30 de setiembre de 2020. El presente texto es una revisión de la versión original. 


\section{Introducción}

E n el XXVI aniversario de la Facultad de Ciencias Sociales de la Universidad Nacional Mayor de San Marcos, que coincide con momentos de suma gravedad por los efectos de la pandemia del COVID-19 y la crisis social en ciernes, es indispensable debatir sobre la importancia del conocimiento social en América Latina.

Reflexionar sobre los grandes problemas teóricos y epistemológicos en la producción de las ciencias sociales es fundamental para examinar la emergencia de un discurso propio latinoamericano frente a la hegemonía de la perspectiva eurocéntrica. Procesos cognoscentes de las ciencias sociales en la región que vienen desenvolviéndose desde las últimas décadas del siglo pasado y con claras manifestaciones internacionales.

De esa forma, se examina el desarrollo de una perspectiva autónoma del conocimiento en América Latina. El desarrollo de un pensar singular que subraya la persistencia por distanciarse críticamente del universalismo abstracto eurocéntrico, repensando las posiciones cognoscitivas y experiencias de la región que confluyen en otro universalismo concreto depositario de todos los saberes particulares.

La exposición presenta algunas consideraciones preliminares que permitan facilitar una discusión mayor frente a los desafíos y posibilidades cognoscitivas de América Latina en el mundo actual.

En ese sentido, el análisis de la senda de reoriginalización cognoscitiva de América Latina advierte la exploración de otro sentido de la historia universal, sostenido en la unidad compleja de las ciencias sociales, la gestación de nuevas problemáticas de investigación y los desarrollos de discursos teóricos alternativos.

\section{Otro sentido de la historia}

En las últimas décadas del siglo pasado "emerge un nuevo debate latinoamericano" y se abre un momento de disputa (Quijano 2014: 11), las ciencias sociales del continente ponen en revisión los mismos fundamentos que sustentan la universalidad de las teorías, los conceptos y las categorías que fueron elaboradas para examinar realidades de Europa y Estados Unidos.

En América Latina las ciencias sociales pugnan por cuestionar y desembarcarse de la forma de conocer eurocéntrica prevaleciente. Forma eurocéntrica de pensar que se impone como la única racionalidad de validez universal del conocimiento porque se configura sobre el desplazamiento y deslegitimación de los saberes locales; basta citar algunos datos, la región solo produce el $4.7 \%$ del conocimiento reconocido mundialmente (RICYT 2018:152) y el $98.7 \%$ de las citaciones mayores son de origen norteamericano (Beigel 2013: 120). Randall Collins (2005) expone la evolución de más de quinientos ańos de las ideas globales, que discurren desde Oriente, Grecia, Roma, el mundo árabe hasta Europa moderna, no hace ninguna mención a los desarrollos intelectuales latinoamericanos, porque se entiende que carecen de las posibilidades teóricamente racionales y los cuales no cumplen con los requerimientos euro-norteamericano. Ahora, se trata de quebrantar ese nudo que ahoga nuestros saberes.

Latinoamérica se transforma en fuente de pensar, imaginar y producir conocimiento social que persigue subrayar la especificidad de nuestro continente (Mejía 2016). Se debate la elaboración de conocimientos desde nuestra peculiar existencia social, persistiendo en la ruta cultural trazada por Guaman Poma de Ayala, José Carlos Mariátegui, Aníbal Quijano y otros. Camino seguido en la región por las "epistemologías del sur" enfatizado principalmente por Boaventura de Sousa Santos, Enrique Dussel y Pablo González Casanova. También expuesto por los viejos maestros de las ciencias sociales peruanas, todos ellos de la Universidad de San Marcos; se pueden destacar las narrativas representativas de Julio C. Tello en la arqueología, Jorge Basadre en la historia, Luis E. Valcárcel en la antropología y Javier Pulgar Vidal en el campo de la geografía. Desde ángulos diferenciados, todos comparten la propensión por indagar un significado de los sucesos históricos latinoamericanos orientados hacia la emancipación de la humanidad.

La relación entre tiempo e historia en la episteme latinoamericana configura un rasgo distintivo, diferenciado del significado expuesto por G. W. F. Hegel en sus Lecciones sobre la filosofía de la historia universal, como derivación del movimiento histórico de las ideas, del espíritu racional, que se objetiva en forma 
absoluta en el devenir de la humanidad: "Europa es absolutamente el término de la historia universal" (2005: 308), todas las demás prácticas societales son reproducciones del camino alcanzado por la modernidad eurocéntrica.

La búsqueda permanente de un pensamiento que permita reelaborar los principios de un sentido propio de la historia latinoamericana, no es el recuento puramente arbitrario de los hechos, al contrario, en forma probabilística los sucesos pueden tener derivaciones significativas de justicia humana, en tanto "son portadores de sentido" y "susceptibles de transformar el sentido de la evolución que desencadenan" (Prigogine y Stengers 1992: 53). En América Latina no solamente se tiene en cuenta su particularidad como parte de una tendencia general, sino, lo más importante, reconstruye conceptos específicos con características típicas y define categorías singulares emergentes del potencial cambio social, en interrelación profunda con las otras experiencias del mismo proceso moderno. Precisamente, porque el sistema capitalista encontró su espacio y tiempo basal en la conquista de América Latina, desde entonces marcó el horizonte histórico de nuestra experiencia de modernidad y, a la vez, condicionó la posibilidad de un camino alternativo. Igualmente, en condiciones que la modernidad global apertura un tiempo histórico de ruptura y bifurcación, irrumpen prácticas cognoscitivas diferenciadas de los modos de pensar e imaginan otras propuestas epistémicas del mundo en relación a valores de libertad, igualdad social y defensa de la vida en la Tierra. En esa configuración, se desplegarían nuevos rumbos de la historia de América Latina.

\section{Unidad de las ciencias sociales}

Una nueva perspectiva cognoscitiva en América Latina se desliza hacia la indagación de un itinerario contradictorio que implique la unidad de las ciencias sociales en tres direcciones interrelacionadas.

Unidisciplinariedad de las ciencias sociales. Viejo sueño que viene desde fines del siglo XIX y que ha ido constituyéndose en la propuesta de Immanuel Wallerstein de la "forma unidisciplinaria" (1979: 18), vinculada a la construcción de una visión de la totalidad social heterogénea que sustenta la reunificación de una "ciencia social histórica" (2004: 138).

La ciencia social unitaria apertura la posibilidad de intentar desarrollarse más allá del monoconocimiento de las disciplinas particulares, admite que el carácter distintivo de los sistemas sociales es multidimensional y, además, consiente sortear un gran vacío cognoscitivo al restituir el significado holístico de la existencia humana.

Unidad con las ciencias naturales. Según la propuesta del Premio Nobel de Química de 1977 Ilya Prigogine, quien expone la "unidad del conocimiento científico" (1990: 12) entre las ciencias sociales, las ciencias naturales y ciencias humanas, definidas por las nociones de un orden alejado del equilibrio y de cambio permanente.

En esa dirección, debido a la sensibilidad de las ciencias sociales o por su semejanza con los ámbitos de las "partículas llamadas elementales y los de la cosmología [que] corresponden a fenómenos sumamente complejos" (Prigogine 1993: 15). Se perfila el diseńo de un "diálogo entre el hombre y con el mundo que habitan" (Prigogine 1990: 353) fundado en actividades de saberes complementarios con una epistemología común de las ciencias de la complejidad.

Unidad con los otros saberes. La conquista de América conllevó el divorcio radical entre los saberes modernos y los nativos, a estos últimos condenándolos al sometimiento y al exterminio. Desde entonces, el eurocentrismo ha tratado de imponer una pretendida homogeneización de la cognición mundial.

José Carlos Mariátegui se opuso firmemente y formuló la unidad del logos y el mito: "Pero ni la Razón ni la Ciencia pueden ser un mito. Ni la Razón ni la Ciencia pueden satisfacer toda la necesidad del infinito que hay en el hombre. La propia Razón se ha encargado de demostrar a los hombres que ella no les basta. Que únicamente el Mito posee la preciosa virtud de llenar su yo profundo" (1987: 22). La razón moderna se muestra limitada para conocer el saber infinito de la sociedad y la naturaleza, el mito admite un amplio campo de saberes indígenas que permiten acercarnos a la biodiversidad, la medicina, lo sísmico, la educación y otros campos del mundo (De Sousa Santos 2018 y Leff 2004). De forma similar, se incluyen los otros conocimientos originados por las demandas de los movimientos de la sociedad en sus propuestas de nuevos sentidos históricos en la 
diversidad ecológica, feminista, juvenil, inmigrante o frente a las desigualdades sociales (Mejía 2019).

\section{Desafíos de la investigación social}

La investigación social en un mundo trazado por la crisis y el cambio profundo personifica nuevos retos y problemáticas que admitan comprender la realidad e instituir las oportunidades de anticipar los fenómenos, con lo cual proporcionan pautas para la crítica y acción futura.

En ese sentido, la investigación social se orienta al examen de las dos principales temáticas que definen la sociedad contemporánea. El primero es el análisis de las desigualdades extremas, generado como en ningún momento de la evolución histórica, únicamente son siete personas las que controlan la mitad de la riqueza producida en el Planeta (Riffkin 2018), los restantes 7,700 millones de personas que se las arreglen como pueden. En un mundo desbocado por la desigualdad cobran centralidad la precariedad, la flexibilidad y el desempleo (Standing 2016); la modernidad neoliberal movida por la revolución tecnológica e inteligencia artificial modifican radicalmente los proceso productivos al limitar el trabajo asalariado estable, es probable que el sistema social siga reproduciéndose en función del desempleo, la informalidad, la pobreza y el trabajo forzado; lo paradójico es que ocurre cuando se genera más empleo y riqueza en diversas áreas del planeta. Las mayorías poblacionales se encuentran inmersas en un proceso de reclasificación como seres inferiores al vaciarlos de sus derechos para ser explotados y si fuera posible desechados, porque a los seres humanos iguales no se les puede subordinar.

Segundo, la investigación social se desliza al estudio de la destrucción de la naturaleza que pone en peligro la vida en la Tierra por la expansión de más de cinco siglos de modernidad, en las últimas décadas llevado a niveles trágicos entre la acumulación ilimitada del capital y los recursos restringidos del planeta. Actualmente tenemos la tasa de mortalidad de seres vivos de 100 veces más alta que la natural (Ceballos et al. 2015), el $25 \%$ de todas las especies de la Tierra se encuentran en peligro de extinción (IPBES 2019) y en el Perú durante los últimos cuarenta años se ha perdido irremediablemente el $42.64 \%$ de los glaciares por el impacto del calentamiento global, con los cambios climáticos y ecológicos respectivos (ANA
2014: 15). La pandemia de Covid-19 que padece el mundo, al parecer es resultado de la destrucción del hábitat de los seres animales, la arremetida de la vida social los arrincona a espacios cada vez más reducidos, condenándolos al exterminio y a la amenaza de que los virus congénitos salten a otros animales $y$, luego, al hombre. Es posible que esta forma de virus haya surgido en el sur de China y de ahí se extendió por el mundo (Ramonet 2020).

La investigación social tiene la imperiosa necesidad de dar cuenta de un mundo moderno que abandonó los grandes logros que trazó originalmente la modernidad, descrito por Bauman (2013) como una "modernidad sin modernismo" o como lo expresara Quijano (2014a) de una "modernidad sin des/colonialidad”. En los últimos tiempos pareciera que hemos ingresado a un momento histórico donde el sistema moderno puede afectar gravemente la existencia de la humanidad y la manifestación de la vida en la Tierra.

\section{Pensamiento latinoamericano}

El pensamiento latinoamericano de las ciencias sociales expresa un discurso sobre las limitaciones del mundo moderno y abre las posibilidades de meditar una sociedad más igualitaria y de defensa de la vida en la naturaleza. Perspectiva de América Latina que tiene varias aristas, se destacan el neodesarrollismo, la teoría crítica, los estudios "étnicos» y la colonialidad del poder.

La nueva teoría crítica. Pensamiento de América Latina que asume los grandes avances de conocimiento contemporáneo, en especial los desarrollos de diversos autores como Pierre Bourdieu, Manuel Castells, Zygmunt Bauman, Noam Chosmky, por señalar algunos (Piedrahita et al. 2015). Discursos que objetan el sistema establecido o algunos de sus aspectos centrales en relación con los principios de justicia social (Altamirano et al. 2009: 14). Sin embargo, cabe destacar por su influencia decisiva en el debate internacional al medioambientalista inglés James Lovelock (2007) por la teoría de la Gaia, quien descubre la ruptura del orden sistémico de la Tierra, fruto de las caóticas relaciones de lo químico, físico, biológico y humano, poniendo en peligro toda forma de vida. A diferencia de otros periodos históricos de fractura del equilibrio fue consecuencia de la misma naturaleza, el 
presente es resultado directo de la acción humana, de ahí su carácter extremadamente destructivo y agresivo. También se destaca la obra de Thomas Piketty (2015) por sus investigaciones concretas de la exorbitante desigualdad social en la modernidad del siglo XXI, que ha develado los sofismas del neoliberalismo, derivación de un capitalismo oligárquico en la que el capital neto familiar tiene un crecimiento mayor al conjunto del país, potenciando la concentración de la riqueza a niveles siderales. Piketty renueva la teoría crítica, viabiliza pensar más allá del sistema moderno capitalista y facilita imaginar otra forma de sociedad más justa.

El neodesarrollismo propugna la presencia central del Estado en la distribución de recursos y el impulso de las capacidades productivas, asumiendo la crítica al neoliberalismo y la reivindicación de los valores locales (García 2013). Pareciera que las experiencias neodesarrollistas en América Latina - Brasil, Venezuela, Ecuador, Bolivia- se encuentra en crisis, en gran parte, por su carácter extractivista, la corrupción del Estado y la desintegración de territorios nativos (Calderón y Castells 2019). En cambio, su mayor éxito se encuentra en el Asia - China, Singapur, Rusia e India_ - por su énfasis en la revolución tecnológica, la industrialización y revalorización de las subjetividades, como el budismo, eslavismo, islamismo, confucionismo (Mignolo 2012). En general, la perspectiva neodesarrollista busca desvincularse del proyecto global neoliberal y mantenerse en la modernización capitalista, anclado en las experiencias culturales regionales.

Los estudios "étnicos", desarrollados en algunos departamentos y asignaturas de las universidades norteamericanas, como la de Berkeley, y la presencia de los "filósofos 'latinos' en Estados Unidos" (Dussel, 2007). La presión de los grupos segregados y discriminados de negros, indígenas, latinos, chicanos, mujeres, chinos a partir de una "epistemología fronteriza" y la necesidad por "re-existir" subrayan la importancia de elaborar un pensamiento propio, como condición indispensable para empoderar reflexivamente a las comunidades racializadas, reforzando sus identidades y ganar libertades (Mignolo 2015). Contribuyendo a desarrollar una crítica al eurocentrismo en el mismo núcleo donde se forjan los significados de la modernidad global y formula una epistémica de libertad de los inmigrantes, la etnicidad, el género y la tolerancia de todas las minorías sociales. Estudios "étnicos" que irrumpen en el pensamiento contemporáneo revisando los contenidos de nación e identidad, cultura, los cuerpos "de color", relaciones de género y decolonialidad (Maldonado-Torres 2006). Expresa una trayectoria epistémica que abre la posibilidad de modificar el carácter de las poblaciones segregadas de simples objetos a sujetos reflexivamente empoderados y activos.

La teoría de la colonialidad del poder, propuesto por Aníbal Quijano (1992, 2014b), instituye el discurso latinoamericano más integral frente al universalismo europeizado ${ }^{2}$. A partir de la conquista de América se genera el patrón de poder moderno que integra dos aspectos imbricados, un proceso de explotación fundado en la hegemonía del capital y otro en la dinámica de dominación basada en la inferiorización de la población que al expropiarles sus derechos y adjurándoles sus capacidades morales los convierten en seres indignos y humillados para revertir su humanidad. Modernidad y colonialidad son aspectos complementarios de un mismo proceso mundial. Patrón de organización de la sociedad que convierte la "vida humana desechable" y, a la vez, la naturaleza en "desechable" para su explotación mercantil creciente, admitiendo la posibilidad de destrucción de la vida en la Tierra. La globalización ha llevado la pérdida de soberanía de los países por la re-privatización del poder en un "bloque imperial mundial" (Quijano 2001), que redefine el papel del Estado-nación por la reconcentración del poder en una élite a escala planetaria. Paralelamente, el proceso de precarización e inferiorización de las mayorías condiciona que los derechos ciudadanos se limiten a las capas más cercanas al poder y el Estado se vaya transformando en una entidad privada y excluyente, por la hegemonía de los mecanismos de «apropiación /violencia» (De Sousa Santos 2010: 20-30). En ese contexto, el eurocentrismo se transforma en el imaginario social, la memoria histórica y la perspectiva de conocimiento que permite organizar todas las subjetividades en un gran discurso universal homogéneo. La colonialidad expresa el encuentro creativo con la rica tradición cognoscitiva contestaria de América Latina, en particular a lo relativo a la opción de la reproducción democrática de la existencia

2 Puede consultarse Quijano (2014b), Palermo y Quintero (2014), Mignolo (2003), Mejía (2014) y Germaná (2010). 
social, la libertad individual y de la defensa de las condiciones de vida en el universo.

Propuestas de América Latina que permiten configurar perspectivas teóricas más allá de la modernidad, donde la razón solo pueda imaginarse con los postulados de una sociedad como parte de la naturaleza, de igualdad social y libertad entre las personas.

\section{A modo de conclusión}

La exposición muestra el largo desarrollo, no exento de contradicciones, por distanciarse del pensamiento eurocéntrico, a partir de la unidad múltiple de las ciencias sociales, impulsando una investigación social centrada en la problemática de las desigualdades sociales y la destrucción de la naturaleza, y la formulación de nuevos discursos teóricos alternos. Favoreciendo la exigencia de reconstruir los fundamentos del conocimiento y de las categorías que permitan significar la realidad específica latinoamericana.

La experiencia de América Latina se revela como una de las fuentes más interesantes del proceso de formación de otra episteme, emancipada de las presiones eurocéntricas y construida como parte del sistema mundo moderno. De esa forma, la importancia de nuestro pensamiento significa el trazo de originalidad para analizar la realidad específica y singular de América Latina, y, al mismo tiempo, expresa un pensar crítico porque pone en evidencia los mecanismos de explotación y dominación del continente.

Los conocimientos sociales latinoamericanos buscan reencontrarse con la vida social, como lo eran en las sociedades nativas prehispánicas (Shady et al. 2019). En la promesa de una ciencia social comprometida con la sociedad por su alta reflexividad, las teorías y conceptos se integran en el tiempo como parte de la vida cotidiana y de las instituciones de la sociedad (Lamo de Espinosa 1990); postulando la responsabilidad de la Investigación Acción (González Casanova 2004) que involucra la participación de las poblaciones en los procesos de investigación y cambio social; y como ciencias sociales aplicadas, dirigidas a la formación de la acción racional en las organizaciones de la sociedad (Gouldmer y Miller 2019, Requena y Ayuso 2016).

En general, la revisión del conocimiento social latinoamericano se expone como un cuestionamiento a la subjetividad moderna, desatada por las mutacio- nes de la globalización neoliberal, cuyos principios envuelven el modo de pensar de la sociedad. En ese sentido, el conocimiento social se relaciona estrechamente al devenir de la modernidad.

\section{Bibliografía}

Autoridad Nacional de Agua (ANA) (2014): Inventario nacional de glaciares y lagunas. Lima: ANA.

Altamirano, C.; De Sousa Santos, B; Torres-Rivas, E. y Miró, C. (2009). "Encuesta sobre el pensamiento crítico en América Latina”. Crítica y Emancipación Revista Latinoamericana de Ciencias Sociales (pp. 9-24). Año I, No 2, Buenos Aires.

Bauman, Z. (2013): La cultura en el mundo de la modernidad líquida. México: FCE.

Beigel, F. (2013): Centro y periferias en la circulación internacional del conocimiento. Nueva sociedad (pp. 110-123), No 245. https://nuso.org/media/articles/ downloads/3944_1.pdf

Calderón, F. y Castells, M. (2019): La nueva América Latina. México: FCE.

Ceballos, G.; Ehrlich, P.; Barnosky, A.; García, A.; Pringle, R. y Palmer, T. (2015): "Accelerated modern human-induced species losses: entering the sixth mass extinction". Science Advances, 1 (5). https://www. transcend.org/tms/wp-content/uploads/2017/07/ Accelerated-modern-human\%E2\%80\%93inducedspecies-losses-Entering-the-sixth-mass-extinction.pdf

Collins, R. (2005): Sociología de las filosofias: una teoría del cambio intelectual. Barcelona: Editorial Hacer.

De Sousa Santos, B. (2018): Construyendo las Epistemologias del Sur: para un pensamiento alternativo de alternativas, V. I. CLACSO: Buenos Aires.

Dussel, E. (2007): Politica de la liberación. Historia mundial critica. Editorial Trotta: Madrid.

García Linera, A. (2013): Democracia, Estado y nación. Vicepresidencia del Estado Plurinacional: La Paz.

Germaná, C. (2010): "Una epistemología otra: El proyecto de Aníbal Quijano”. Nomadas (pp. 77-94). № 32: Bogotá.

González Casanova, P. (2004). Las nuevas ciencias y las humanidades. De la academia a la politica. Barcelona: Anthropos - IIS de la UNAM - Editorial Complutense. Gouldmer, A. y Miller, S.M. (2019): Sociología aplicada. Problemas y oportunidades. Madrid: CIS.

Hegel, G. W. F. (2005): Lecciones sobre la filosofía de la historia universal. Madrid: Tecnos. 
Lamo de Espinosa, E. (1990): La sociedad reflexiva. Sujeto y objeto del conocimiento sociológico. Madrid: Siglo XXI- CIS.

LEFF, E. (2004): Racionalidad ambiental. La reapropiación de la naturaleza. México: Siglo XXI.

Lovelock, J. (2007): La venganza de la tierra. La teoría de la Gaia y el futuro de la humanidad. Barcelona: Planeta.

Maldonado-Torres, N. (2006): "Pensamiento crítico desde la sub-alteridad: los estudios étnicos como ciencias descoloniales o hacia la transformación de las humanidades y las ciencias sociales" en el siglo veintiuno". Revista Interamericana de Educación de Adultos CREFAL (pp. 143-165), №1: México.

MARIÁTEGUI, J. C. (1987): El alma matinal y otras estaciones del hombre de hoy. Amauta: Lima.

Mejía, J. (2019): "Nuevo horizonte de sentido histórico, crisis de la modernidad y movimientos de la sociedad. El legado teórico de Aníbal Quijano”. Discursos del Sur (pp. 95-109), № 3. UNMSM: Lima.

Mejía, J. (2016): América Latina, modernidad y conocimiento. El desarrollo de otro discurso epistémico. UNMSM: Lima.

Mejía, J. (2014): Colonialidad y des/colonialidad en América Latina. Elementos teóricos. Aníbal Quijano: Des/colonialidad y bien vivir. Un nuevo debate en América Latina. Editorial URP: Lima

Mignolo, W. (2015): Habitar la frontera: sentir y pensar la descolonialidad (Antología, 1995-2014). CIDOB: Barcelona

Mignolo, W. (2012): "Hacia la cartografía de un nuevo mundo: pensamiento descolonial y desoccidentalización (un diálogo con Walter Mignolo). Entrevista realizada por Francisco Carballo". Otros logos. Revista de Estudios Críticos (pp. 237-264), No3. Neuquen: Universidad Nacional de Camahue.

Mignolo, W. (2003): Historias locales, diseños globales. Colonialidad, conocimientos subalternos y pensamiento fronterizo. Madrid: Akal.

Palermo, Z. y Quintero, P. (2014): Aníbal Quijano. Textos de fundación. Buenos Aires: Ediciones del Signo.
Piedrahita, C., Díaz, A. y Vommaro, P. (Compiladores) (2015): Pensamientos críticos contemporáneos: Análisis desde Latinoamérica. Bogotá: Universidad Distrital Francisco José de Caldas - CLACSO.

PiketTy, T. (2015): El capital del siglo XXI. México, FCE.

Prigogine, I. (1993): Las leyes del caos. Barcelona: Crítica.

Prigogine, I. y Stengers, I. (1992): Entre el tiempo y la eternidad. Madrid: Alianza.

Prigogine, I. y Stengers, I. (1990): La nueva alianza. Metamorfosis de la ciencia. Madrid: Alianza.

Quijano, A. (2014a): Des/colonialidad y bien vivir. Un nuevo debate en América Latina. Lima: URP.

Quijano, A. (Antología esencial) (2014b): Cuestiones y horizontes. De la dependencia histórico-estructural a la colonialidadldescolonialidad del poder. Buenos Aires: CLACSO.

Qujjano, A. (1992): "Colonialidad y modernidad/racionalidad”. Perú Indígena (pp. 11-20), Vol.13, No 29: Lima.

Shady, R.; Novoa, P. y Quispe, E. (2019): Los valores de la civilización Caral: Reflexiones para el "buen vivir". Lima: Zona Arqueológica Caral.

Standing, G. (2016): "El precariado y la lucha de clases". Sin Permiso: República y socialismo también para el siglo $X X I$, No 15 (Segunda Época), pp. 87-102.

RAMONET, I. (2020): "La pandemia y el sistema-mundo". La Jornada, 25 de abril: México. https://www.jornada. com.mx/ultimas/mundo/2020/04/25/ante-lo-desconocido-la-pandemia-y-el-sistema-mundo-7878.html

Red de Indicadores de Ciencia y Tecnología -Iberoamericana e Interamericana- (RICYT) (2018): "El estado de la ciencia. Principales indicadores de ciencia y tecnología iberoamericanos / interamericanos". http://www.ricyt.org/2018/10/ el-estado-de-la-ciencia-2018/

Requena, F. y Ayuso, L. (2016): Teoría sociológica aplicada. Madrid: Anthropos.

Rifkin, J. (2018): La tercera revolución industrial. Santa Fe Debates. https://www.youtube.com/ watch?v=5E7oAYefZss

Wallerstein, I. (2004): Las incertidumbres del saber. Barcelona: Gedisa.

WaLLERSTEIn, I. (1979): El moderno sistema mundial I. La agricultura capitalista y los origenes de la economía europea en el siglo XVI. Madrid: Siglo XXI. 